\title{
The relationship between lymphedema severity and awareness of lymphedema surgery
}

\author{
Hyun Seung Lee ${ }^{1}$, Yong Chan Bae ${ }^{1,2}$, Su Bong Nam ${ }^{1,2}$, Chang Ryul Yi ${ }^{1,2}$, Jin A Yoon ${ }^{2,3}$, \\ Joo Hyoung $\mathrm{Kim}^{1,2}$ \\ ${ }^{1}$ Department of Plastic and Reconstructive Surgery, Pusan National University School of Medicine, Busan; ${ }^{2}$ Biomedical Research Institute, \\ Pusan National University Hospital, Busan; ${ }^{3}$ Department of Rehabilitation Medicine, Pusan National University School of Medicine, Busan, \\ Korea
}

\begin{abstract}
Background During the early stages of lymphedema, active physiologic surgical treatment can be applied. However, lymphedema patients often have limited knowledge and misconceptions regarding lymphedema and surgical treatment. We analyzed the correlations between lymphedema severity and surgical technique according to patients' awareness of surgical treatment for secondary upper extremity lymphedema (UEL).
\end{abstract}

Methods Patients with UEL diagnosed between December 2017 and December 2019 were retrospectively evaluated. At the time of their presentation to our hospital for the treatment of lymphedema, they were administered a questionnaire about lymphedema and lymphedema surgery. Based on the results, patients were classified as being aware or unaware of surgical treatment. Lymphedema severity was classified according to the arm dermal backflow (ADB) stage and the MD Anderson Cancer Center (MDACC) stage based on indocyanine green lymphography conducted at presentation. Surgical techniques were compared between the two groups.

Results Patients who were aware of surgical treatment had significantly lower initial ADB and MDACC stages $(P<0.05)$ and more frequently underwent physiologic procedures than excisional procedures $(P=0.003)$.

Conclusions If patients are actively educated regarding surgical treatment of lymphedema, physiologic procedures may be performed during the early stages of UEL.

Keywords Breast cancer lymphedema / Upper extremity / Lymphography / Awareness

\author{
Correspondence: Joo Hyoung Kim \\ Department of Plastic and \\ Reconstructive Surgery, Pusan \\ National University School of \\ Medicine, 179 Gudeok-ro, Seo-gu, \\ Busan 49241, Korea \\ Tel: +82-51-240-7273 \\ Fax: +82-51-243-9405 \\ E-mail: medic144@hanmail.net
}

\section{INTRODUCTION}

Lymphedema is a progressive disease affecting the lymphatic system involving chronic inflammation, fibrosis, hyperkeratosis, and adipose deposition [1]. It is mostly observed following oncological treatment, such as radiotherapy or lymph node dissection [2-4]. Secondary lymphedema after breast cancer surgery occurs in up to $30 \%$ of patients following axillary lymph node dissection [5]. Lymphedema results in significant pain and morbidity, and it negatively impacts patients' quality of life. It also results in a significant financial burden for patients and the healthcare system [6]. Therefore, active support and treatment for patients with lymphedema are required.

Complete decongestive therapy is the standard of care for 
lymphedema patients. However, surgical treatment has recently become increasingly popular $[7,8]$. Surgical treatment can be classified as excisional and physiologic procedures. Despite the promising short-term outcomes of excisional surgical techniques and their ability to yield long-term reductions in limb circumference, these techniques cannot reverse the underlying pathophysiological processes of lymphedema, and patients are frequently required to maintain lifelong adherence to complete decongestive therapy and compression therapy [9]. In contrast, advanced microsurgical procedures, such as lymphovenous anastomosis (LVA) and vascularized lymph node transfer (VLNT), which are physiologic procedures, aim to improve lymphatic drainage via physiologic mechanisms [9] and to reverse the pathophysiological processes of the disease. Therefore, it is important to perform active surgical treatment during the early stages of lymphedema, when physiologic procedures are possible.

It has been reported that patients have difficulty finding and/ or understanding health information concerning lymphedema and its surgical management [10]. Additionally, most patients have been reported to learn about lymphedema surgery from sources who are not lymphedema specialists and, therefore, have an insufficient knowledge of lymphedema and its treatment [11]. Thus, patients who do not have sufficient and accurate knowledge of lymphedema and its surgical treatment are unlikely to undergo surgical treatment during the early stages of the disease.

This study analyzed the relationship between patients' awareness of lymphedema surgery and lymphedema severity at presentation. It also assessed whether patients' knowledge of surgical treatment for lymphedema was associated with their initial presentation to a hospital at an earlier stage of lymphedema, resulting in earlier surgical treatment.

\section{METHODS}

\section{Patients}

Approval from the Institutional Review Board of Pusan National University Hospital (IRB No. 2009-013-095) was obtained before conducting this retrospective study. Fifty-two patients who were referred to our hospital for the assessment of upper extremity lymphedema (UEL) between December 2017 and December 2019 were initially included if they met the following criteria: (1) completed a survey about lymphedema and surgical treatment of lymphedema at presentation and (2) underwent indocyanine green (ICG) lymphography for the evaluation of UEL at presentation. The following patients were excluded: those who refused to complete the questionnaire sur- vey; those with previous primary lymphedema; those lost to follow-up after lymphedema surgery; those with metastasis or infection of both arms; and those in whom staging was not possible due to atypical findings or poor image quality.

All patients were asked to complete a survey designed to assess their awareness of lymphedema and lymphedema surgery, as well as their source of knowledge and the information they obtained about lymphedema surgery when they visited Pusan National University Hospital for the treatment of lymphedema (Fig. 1). Based on their responses, patients were classified as being aware or unaware of surgical treatment. Moreover, at the time of presentation, information was obtained on patients' history of radiotherapy, adjuvant chemotherapy, neoadjuvant chemotherapy, and surgery, including axillary lymph node dissection, mastectomy, and breast-conserving surgery.

\section{ICG lymphography}

We obtained ICG lymphography images using a near-infrared camera (Moment K; IANC\&S, Seoul, Korea) with a 760-nm light-emitting diode and a filter that limited light to $<820 \mathrm{~nm}$. All patients were subcutaneously injected with $0.2 \mathrm{~mL}$ of ICG (Diagnogreen, $2.5 \mathrm{mg} / \mathrm{mL}$ ) into the first and third web spaces of the hand. To reduce pain, $2 \%$ lidocaine $\mathrm{HCl}$ with $1: 100,000$ epinephrine was injected into the first and third web spaces of the hand before dye injection. After ICG dye injection, images of the anterior and posterior aspects of the lymphatic drainage of both upper limbs were obtained using a near-infrared camera. ICG lymphography images were obtained hourly post-injection until the ICG dye spread throughout the entire limb and was sufficient for ICG lymphography staging.

The images were classified according to the arm dermal backflow (ADB) stage [12] and MD Anderson Cancer Center (MDACC) stage [13] based on the dermal back flow pattern and its extent. Scoring for the ADB stage was performed using the posterior aspect. ICG lymphography staging was conducted in a blinded manner, without information regarding patients' clinical status.

\section{Surgical techniques}

LVA, VLNT, and power-assisted liposuction were performed to treat lymphedema. The surgical technique applied for each patient was determined based on the clinical evaluation, ICG lymphography results, and lymphoscintigraphy findings. If the severity of lymphedema was low, then LVA and VLNT were predominantly considered; liposuction was performed if the severity was high. Patients were asked to supply compression garments or compression bandages, which were placed immediately after LVA, VLNT, and liposuction. Patients were asked to 


\section{Questionnaire about awareness of lymphedema surgery}

Cautions: The following survey is intended to assess patients' understanding of lymphedema surgery. Please read each question carefully and answer the following questions. Your responses will be kept confidential and combined with other responses when reporting the results. Please do not write your name on this questionnaire.

1) What is your sex?
( ) Male
( ) Female

2) How old are you?

( )

3) What is your highest level of education?
( ) Less than high school
( ) High school graduate
( ) College graduate
( ) Postgraduate school

4) How long have you been suffering from lymphedema?

( ) Less than 6 months

( ) Between 6 months and 1 year

( ) Between 1 and 2 years

( ) Between 2 and 5 years

( ) More than 5 years

5) Did you know about the surgical treatment of lymphedema when you visited the tertiary hospital (Pusan National University Hospital) for the treatment of lymphedema?

( ) Yes
6) How long after being diagnosed with lymphedema did you learn about lymphedema surgery?

( ) After visiting Pusan National University Hospital for the treatment of lymphedema

( ) At the time of diagnosis/before diagnosis

( ) Less than 6 months

( ) Between 6 months and 1 year

( ) Between 1 and 2 years after

( ) Between 2 and 5 years

( ) More than 5 years

If you choose "after visiting Pusan National University Hospital for the treatment of lymphedema" skip question number 6 .

7) How did you first learn about lymphedema surgery?

( ) From a physician

( ) From a health care professionals

( ) From family/friend

( ) Internet
8) Which treatments have you previously received for lymphedema? Please select all that apply.

( ) Compression bandage

( ) Physical or occupational therapy

( ) Medications

If medications, please describe;

If other, please describe;

9) Have you had any surgery for reasons other than lymphedema?

( ) Yes

( ) No

If yes, please list all previous surgeries;

Fig. 1. Questionnaire about the patients' awareness of lymphedema surgery. Patients were asked to complete a questionnaire designed to evaluate their awareness of lymphedema and lymphedema surgery.

wear compression garments or compression bandages for approximately 6 months postoperatively.

\section{Statistical analysis}

Values are presented as mean \pm standard deviation or as percentages when appropriate. The Wilcoxon rank-sum test was performed for continuous variables. The Fisher exact test was performed for categorical variables. All data were analyzed using the R 4.0.1 IRR package (R Foundation for Statistical Computing, Vienna, Austria), and a P-value $<0.05$ was considered to indicate statistical significance.

\section{RESULTS}

Of the 52 patients who were referred to our hospital for UEL assessment during the study period, 48 were finally included in the study. No patient was excluded from the analysis due to poor imaging quality results of the final test. However, one patient refused to complete the survey, and another patient was excluded because of bilateral UEL. Two other patients were excluded because they were diagnosed with primary lymphedema.

Demographic characteristics were compared between patients who were aware or lymphedema surgery $(n=34)$ and those who were not $(n=14)$ (Table 1). All patients were female and had a history of breast cancer. The mean age of those who were aware of lymphedema surgery was $55.09 \pm 8.01$ years, while that of those who were not aware of lymphedema surgery was 57.29 \pm 10.97 years. This difference was not statistically significant $(\mathrm{P}=0.506)$. Preoperative and postoperative body mass index (BMI), limbs with lymphedema, and educational levels were likewise not significantly different between the two groups. No significant between-group difference was found in the duration of suffering from lymphedema $(\mathrm{P}=0.458)$. Preoperative and postoperative BMI values were calculated based on the height and weight measured at presentation and height and weight measured at the time of the outpatient visit at 6 months after surgery, respectively. The proportions of patients with a history of radiotherapy, adjuvant and neoadjuvant chemotherapy, and 
Table 1. Patient demographics

\begin{tabular}{|c|c|c|c|c|}
\hline Characteristics & Total & Aware of lymphedema surgery & Unaware of lymphedema surgery & P-value $e^{a)}$ \\
\hline No. of patients & 48 & 34 & 14 & \\
\hline Female sex ${ }^{b)}$ & $48(100.00)$ & $34(100.00)$ & $14(100.00)$ & \\
\hline Age (yr) & $55.73 \pm 8.91$ & $55.09 \pm 8.01$ & $57.29 \pm 10.97$ & 0.506 \\
\hline \multicolumn{5}{|l|}{ Body mass index $\left(\mathrm{kg} / \mathrm{m}^{2}\right)$} \\
\hline Preoperative & $24.73 \pm 2.72$ & $24.65 \pm 2.90$ & $24.92 \pm 2.29$ & 0.732 \\
\hline Postoperative & $24.60 \pm 2.80$ & $24.56 \pm 3.05$ & $24.71 \pm 2.18$ & 0.851 \\
\hline Affected limbs & & & & 1.000 \\
\hline Right & $21(43.8)$ & $15(44.1)$ & $6(42.9)$ & \\
\hline Left & 27 (56.2) & $19(55.9)$ & $8(57.1)$ & \\
\hline Education & & & & 0.953 \\
\hline Less than high school & $5(10.4)$ & $3(8.8)$ & $2(14.3)$ & \\
\hline High school graduate & $20(41.7)$ & $14(41.2)$ & $6(42.9)$ & \\
\hline College graduate & $20(41.7)$ & $15(44.1)$ & $5(35.7)$ & \\
\hline Postgraduate school & $3(6.2)$ & $2(5.9)$ & $1(7.1)$ & \\
\hline Duration of suffering from lymphedema & & & & 0.458 \\
\hline$<6 \mathrm{mo}$ & $1(2.08)$ & $1(2.94)$ & 0 & \\
\hline $6 \mathrm{mo}$ to $<1 \mathrm{yr}$ & $1(2.08)$ & $1(2.94)$ & 0 & \\
\hline 1 to $<2 \mathrm{yr}$ & $9(18.75)$ & 7 (20.59) & $2(14.29)$ & \\
\hline 2 to $<5 \mathrm{yr}$ & $28(58.33)$ & $21(61.76)$ & $7(50.00)$ & \\
\hline$\geq 5 \mathrm{yr}$ & $9(18.75)$ & $4(11.76)$ & $5(35.71)$ & \\
\hline Radiotherapy & & & & 1.000 \\
\hline No & 9 (18.75) & $6(17.65)$ & $3(21.43)$ & \\
\hline Yes & $39(81.25)$ & $28(82.35)$ & $11(78.57)$ & \\
\hline Adjuvant or neoadjuvant chemotherapy & & & & 0.728 \\
\hline No & $13(27.08)$ & $10(29.41)$ & $3(21.43)$ & \\
\hline Yes & $35(72.92)$ & $24(70.59)$ & $11(78.57)$ & \\
\hline Axillary lymph node dissection & & & & 1.000 \\
\hline No & $3(6.25)$ & $2(5.88)$ & $1(7.14)$ & \\
\hline Yes & $45(93.75)$ & $32(94.12)$ & $13(92.86)$ & \\
\hline Type of surgery & & & & 1.000 \\
\hline Modified radical mastectomy & $9(18.75)$ & 7 (20.59) & $2(14.29)$ & \\
\hline Mastectomy & $36(75.00)$ & $25(73.53)$ & $11(78.57)$ & \\
\hline Breast-conserving surgery & $3(6.25)$ & $2(5.88)$ & $1(7.14)$ & \\
\hline
\end{tabular}

Values are presented as the number (\%) or mean \pm SD.

${ }^{a}$ Wilcoxon rank-sum test for continuous variables and Fisher exact test for categorical variables; ${ }^{b}$ All patients were female.

surgery including axillary lymph node dissection, modified radical mastectomy, mastectomy, and breast-conserving surgery were not significantly different between the groups (Table 1).

All patients in both groups used compression bandages or garments. Fourteen patients who were aware of lymphedema surgery underwent physical or occupational therapy, while this was the case for seven patients who were not aware of lymphedema surgery. Venous circulation improvers and vascular stiffeners were used by seven patients who were aware of lymphedema surgery and four patients who were not. The treatments previously received for lymphedema were similar between the two groups. All patients underwent breast cancer surgery. Other surgical procedures, including cesarean delivery, laparoscopic appendectomy, and carpal tunnel surgery, were not directly associated with the development of lymphedema.

Patients who indicated that they were aware of lymphedema surgery completed additional survey questions, and their responses were assessed (Table 2). Almost half of the patients learned about the availability of surgical treatment 1 to 2 years after diagnosis (41.2\%). Furthermore, they learned about surgical treatment from their physicians $(64.7 \%)$, other healthcare professionals (26.5\%), or the internet (8.8\%). Among the patients who were aware and unaware of lymphedema surgery, the time intervals from diagnosis to lymphedema surgery were 1 to 2 years for $32.3 \%$ and $28.6 \%$ and 2 to 5 years for $32.3 \%$ and $42.9 \%$, respectively (Table 2). The time interval from diagnosis to lymphedema surgery was not significantly different between the two groups $(\mathrm{P}=0.764)$.

To compare the ICG lymphography stage at presentation according to patients' awareness of lymphedema surgery, the $\mathrm{ADB}$ stage and MDACC stage were used. There were significant differences in the staging of the two groups for both systems were 
Table 2. Time interval between diagnosis and awareness of lymphedema surgery and time interval between diagnosis and lymphedema surgery

\begin{tabular}{lcc}
\hline Characteristics & Aware of lymphedema surgery & Unaware of lymphedema surgery \\
\hline Time interval from diagnosis of lymphedema to awareness of & & \\
lymphedema surgery & $4(11.8)$ \\
At the time of diagnosis/before diagnosis & $5(14.7)$ \\
$<6$ mo & $4(11.8)$ \\
6 mo to $<1$ yr & $14(41.2)$ \\
1 to $<2$ yr & $5(14.7)$ \\
2 to $<5$ yr & $2(5.9)$ \\
$\geq 5$ yr & & \\
First source of knowledge about lymphedema surgery & $22(64.7)$ \\
Physician & $9(26.5)$ & \\
Healthcare professional & 0 & \\
Family/friend & $3(8.8)$ & $1(7.1)$ \\
Internet & $4(11.8)$ & $1(7.1)$ \\
Time interval from diagnosis of lymphedema to surgery for lymphedema & $6(17.6)$ & $4(28.6)$ \\
$<6$ mo & $11(32.3)$ & $6(42.9)$ \\
6 mo to $<1$ yr & $11(32.3)$ & $2(14.2)$ \\
1 to $<2$ yr & $2(5.9)$ & 0.764 \\
2 to $<5$ yr & & \\
$\geq 5$ yr &
\end{tabular}

Values are presented as the number (\%).

a)Fisher exact test for categorical variables.

Table 3. Relationship between awareness of lymphedema surgery and ICG lymphography severity stage

\begin{tabular}{lccc}
\hline & Total & Aware of lymphedema surgery & Unaware of lymphedema surgery \\
\hline Arm dermal backflow stage & & & 0 \\
I & $1(2.1)$ & $1(2.9)$ & $1(7.1)$ \\
II & $10(20.8)$ & $9(26.5)$ & $3(21.4)$ \\
III & $19(39.6)$ & $16(47.1)$ & $5(35.7)$ \\
IV & $9(18.8)$ & $4(11.8)$ & $5(35.7)$ \\
V & $9(18.8)$ & $4(11.8)$ & $1(7.1)$ \\
MD Anderson Cancer Center stage & & & $1(7.1)$ \\
I & $2(4.2)$ & $1(2.9)$ & $5(35.7)$ \\
II & $13(27.1)$ & $12(35.3)$ & $7(50.0)$ \\
III & $22(45.8)$ & $17(50.0)$ & 0.013 \\
IV & $11(22.9)$ & $4(11.8)$ & \\
\hline
\end{tabular}

Values are presented as the number (\%).

ICG, indocyanine green.

a) The Fisher exact test was performed, $P<0.05$.

Table 4. Relationships between awareness of lymphedema surgery and surgical techniques

\begin{tabular}{|c|c|c|c|c|}
\hline & Total & Aware of lymphedema surgery & Unaware of lymphedema surgery & P-value ${ }^{\text {a) }}$ \\
\hline Surgical technique & & & & 0.003 \\
\hline \multicolumn{5}{|l|}{ Physiologic procedure } \\
\hline Lymphovenous anastomosis & $36(75.00)$ & $29(85.29)$ & $7(50.00)$ & \\
\hline Vascularized lymph node transfer & $5(10.42)$ & $4(11.76)$ & $1(7.14)$ & \\
\hline \multicolumn{5}{|l|}{ Excisional procedure } \\
\hline Power-assisted liposuction & $7(14.58)$ & $1(2.94)$ & $6(42.86)$ & \\
\hline
\end{tabular}

Values are presented as the number (\%).

${ }^{a}$ Fisher exact test for categorical variables, $\mathrm{P}<0.05$.

used (Table 3). Using both the ADB and MDACC staging systems, the proportion of patients with relatively low stages was significantly higher among those who were aware of lymphede- ma surgery than among those who were not $(P=0.036$ and $\mathrm{P}=0.013$, respectively).

The surgical techniques used in both groups were compared 
(Table 4), and a significant difference was observed $(\mathrm{P}=0.003)$. The overwhelming majority $(97.05 \%)$ of patients who were aware of lymphedema surgery underwent LVA and VLNT, while $2.94 \%$ of patients underwent liposuction. In contrast, $42.86 \%$ of patients who were not aware of lymphedema surgery underwent liposuction, while the remaining $57.14 \%$ underwent LVA and VLNT.

\section{DISCUSSION}

Many risk factors are associated with UEL related to breast cancer, which is quite concerning for patients and their healthcare providers. Furthermore, the risk factors for lymphedema are multifactorial and ambiguous. In this study, the authors aimed to assess patient-related risk factors such as age, sex, BMI, and the affected limb. The history of treatment procedures, such as radiotherapy, adjuvant and neoadjuvant chemotherapy, axillary lymph node dissection, extensive breast surgery, and mastectomy, which are risk factors for lymphedema, was also assessed $[14,15]$. No statistically significant differences in the distributions of these risk factors were observed between groups. However, assessing patients' lifestyle factors, such as income, occupation, hand dominance, and excessive use of the affected limb [16], which may have additional effects, would have helped improve the demographic data analysis.

Lymphedema severity assessed using ICG lymphography images was significantly different between groups; however, the duration of suffering from lymphedema did not show a statistically significant difference. Therefore, the criteria for suffering and perception of suffering may have been ambiguous and may have differed among patients. In this study, patients were instructed that suffering referred to the swelling of the upper limbs, heaviness, tightness, aching, discomfort, motion restriction, recurring infection, and thickening of the skin. Moreover, although statistically insignificant, the proportion of patients who experienced suffering for $>5$ years because of lymphedema was $35.71 \%$ among those who were not aware of lymphedema surgery, which was higher than the proportion observed in those who were aware of lymphedema surgery $(11.76 \%)$. However, further statistical analyses are necessary to better understand the implications of the finding that patients who were unaware of lymphedema surgery experienced suffering for longer periods.

No significant difference was found in the time interval between the diagnosis of lymphedema and surgery (Table 2). However, the percentage of patients who underwent surgery after 2 years was higher among those who were unaware of lymphedema surgery $(57.1 \%)$ than among those who were aware $(38.2 \%)$. In particular, the percentage of patients who received surgery after 5 years was $14.2 \%$ among those who were unaware of lymphedema surgery and 5.9\% among those who were aware. The fact that patients who were unaware of lymphedema surgery constituted a minority of the sample may help explain the lack of statistical significance for this trend. Most patients were diagnosed based on a clinical evaluation; hence, the diagnosis time may have varied between patients depending on the physician's awareness and competence. In addition, patients with only edema - that is, lacking other signs and symptoms associated with lymphedema, such as infection, motion restriction, and tightness - may have delays in visiting the hospital, which in turn delays the diagnosis. These circumstances may be an obstacle in the statistical analysis. Somewhat fewer than half $(41.2 \%)$ of patients who were aware of lymphedema surgery learned about this option 1 to 2 years post-diagnosis, while $20.6 \%$ did so 2 years or more after their diagnosis (Table 2). Earlier awareness of the surgical treatment option earlier may enable more prompt surgery, thereby reducing the time interval between diagnosis and surgery.

We further investigated the reasons for the presentation of patients who were unaware of surgical treatment for lymphedema. Two patients presented to the hospital for wound treatment of the affected limbs, five patients presented to the hospital to undergo rehabilitation for lymphedema and treatment consultation at the department of rehabilitation medicine, and seven patients who had edema in their arm after breast cancer surgery visited the department of general surgery or thoracic surgery and were subsequently referred to a plastic surgery specialist by a general surgery specialist or a thoracic surgery specialist. All of the unaware patients reported that they had not been informed about surgical treatment before they visited the tertiary hospital, despite undergoing rehabilitation treatment for lymphedema. Lymphedema is one of the most devastating complications following surgery for breast cancer $[17,18]$; however, these findings can be interpreted as indicating that many physicians are still unaware of the surgical treatment options available for lymphedema. Therefore, information regarding surgical treatment options for lymphedema should be introduced to physicians to provide comprehensive information to their patients.

Compression decongestive therapy is commonly applied in minor cases of lymphedema when lymphatic dysfunction is seen on ICG lymphography, but edema is not observed clinically. It is also applied when edema is reversible, as established by observing that the swelling decreases when the affected limb is elevated. Surgical procedures are commonly performed if no improvement is observed after 6 months of compression decongestive therapy. However, in this study, several patients who 
were aware of surgical treatment mistakenly thought that it was the last resort. Therefore, quite a few patients who presented to the hospital after the affected limb hardened, after pain was experienced in the affected limb, or when irreversible edema developed in the affected limb. Lymphatic dysfunction leads to fluid stasis in the interstitial area, which initially causes edema. When lymphatic dysfunction causes inflammation, adipose tissue hypertrophy and progressive fibrosis occur. Therefore, it is critical to perform physiologic procedures during the early stages of lymphedema when the adipose tissue has not yet become fibrotic [19]. Fahradyan et al. [11] reported that introducing patients to surgical treatment early during the course of the disease and informing them of surgical options enabled early discussions about lymphedema surgery and that such steps had positive effects on treatment outcomes and quality of life. Physicians must be informed of the surgical treatment options and should be aware that surgical treatment can be performed during the early stages of lymphedema; therefore, these options can be discussed with patients as early as possible.

Clinical evaluations and physical examinations are helpful when diagnosing lymphedema. Nevertheless, as reported by Maclellan et al. [20], 25\% of patients who were referred to a lymphedema specialist did not have true lymphedema; therefore, such evaluations may be inaccurate. Objective diagnostic imaging modalities, including lymphoscintigraphy, magnetic resonance lymphography, and ICG lymphography, are important for evaluating lymph function and distinguishing lymphogenic edema from non-lymphogenic edema. In this study, ICG lymphography, which allows for the safe and immediate evaluation of small lymphatic vessels, was used to classify the lymphedema severity stage. ICG lymphography is more sensitive for detecting early-stage lymphedema, and finer visualization of functional superficial lymphatic drainage can be achieved [21]. However, ICG lymphography has limitations when used to examine deep lymphatic vessels. In our study, lymphoscintigraphy was performed for all patients at 1 to 2 months after presentation to the hospital; however, the results did not reflect those observed at presentation. Therefore, these results were excluded from the study.

The two ICG lymphography stages are generally classified as (1) visible superficial lymphatic vessels and dominant dermal backflow and (2) reflux of lymph fluid into dermal lymphatics. In $\mathrm{ADB}$ staging, the stages are classified according to the extended area of the dermal backflow, while the MDACC staging system classifies cases according to the number of patent lymphatics. Two-stage ICG lymphography, which was used for this study, is a useful method of assessing lymphedema severity. In the present study, patients who were aware of lymphedema sur- gery had significantly lower severity according to both systems than those who were unaware of lymphedema surgery. Regarding the overall staging distribution, $50 \%$ of the patients who were unaware of lymphedema surgery had stage IV lymphedema, while $50 \%$ of the patients who were aware of lymphedema surgery had stage III lymphedema, indicating that less severe lymphedema predominated among those who were aware of the surgical treatment option.

Using the ADB staging system, scoring was performed based on the posterior aspect rather than the anterior aspect of the upper limb because the posterior aspect depends on gravity more than the anterior aspect, thus allowing ICG to spread more widely and making the extended area of the dermal backflow more visible. Additionally, in our previous study, the lymphoscintigraphy severity stage [22] was more positively correlated with the $\mathrm{ADB}$ stage of the posterior aspect than that of the anterior aspect [23]. Therefore, scoring was performed based on the posterior aspect rather than the anterior aspect in this study to obtain a more accurate evaluation of dermal backflow.

There is no universally accepted algorithm to guide decisionmaking regarding the timing, selection, and possible combinations of the available surgical procedures for lymphedema. In this study, patients with high lymphedema severity, as assessed by ICG lymphography performed at the time of presentation, underwent power-assisted liposuction. Patients with a high lymphedema stage included those with $\mathrm{ADB}$ stage $\mathrm{V}$, a small number of patients with $\mathrm{ADB}$ stage IV, a small number of patients with MDACC stage IV, and patients in whom ICG did not move proximally from the injection site. LVA was performed for patients with MDACC stages II and III and for some patients with MDACC stage IV whose superficial patent lymphatic vessels were identified by ICG lymphography. VLNT was considered if a functional lymphatic channel or node was not identified with no or mild upper limb fibrosis. However, the surgical procedures were partially changed depending on patients' clinical symptoms, arm circumference, bioimpedance, and radiological imaging results. Patients who were unaware of lymphedema surgery had larger circumference differences and higher ratios of bioimpedance values between both arms, meaning that this group had more cases of chronic non-pitting lymphedema. Therefore, although the lymphography stages were terminal in both groups, more patients who were not aware of lymphedema surgery had undergone excisional procedures than their counterparts who were aware of surgical treatment options. In addition, all patients received an explanation about all lymphedema surgical procedures, and the final surgical procedure was selected by the patient. Many patients who were aware of lymphedema surgery upon presentation chose physio- 
logic procedures first and proceeded with excisional procedures in the event of unsatisfactory effects of the physiologic procedures; however, the patients who were not aware of lymphedema surgery complained of daily discomfort due to the thickening circumference of their arms and requested an immediate reduction in volume. Therefore, most of the patients who were initially unaware of lymphedema surgery chose excisional procedures rather than physiologic procedures. All patients were followed up for approximately 6 months to 1 year after surgery; if there were no improvements after surgery, liposuction or additional LVA and VLNT were considered.

Our study had some limitations. First, the two groups of patients, defined according to whether they were aware or unaware of lymphedema surgery, had unequal numbers of patients, and the total sample size was small. Although statistical significance may be observed with this sample size, further evaluations involving a larger number of patients are required because only one or two patients were distributed in each subgroup. Second, the questionnaire used to evaluate the patients' awareness of lymphedema and surgery was not validated; however, our findings are valuable in terms of initiating a discussion about patients' awareness of lymphedema surgery and comparing the patients' lymphedema staging and surgical techniques according to their awareness of surgical treatment options.

Patients who were aware of surgical treatment presented to the hospital during earlier stages of lymphedema. Furthermore, these patients were able to actively undergo physiologic procedures such as LVA and VLNT during the early stages of lymphedema. Therefore, promptly and actively providing surgical treatment education to guide patients may facilitate performing physiologic procedures during the early stages of UEL.

\section{NOTES}

\section{Conflict of interest}

Su Bong Nam is an editorial board member of the journal but was not involved in the peer reviewer selection, evaluation, or decision process of this article. No other potential conflicts of interest relevant to this article were reported.

\section{Ethical approval}

The study was approved by the Institutional Review Board of Pusan National University Hospital (IRB No. 2009-013-095) and performed in accordance with the principles of the Declaration of Helsinki. Written informed consent was obtained.

\section{Author contribution}

Conceptualization: JH Kim. Data curation: HS Lee. Formal analysis: HS Lee, JA Yoon. Methodology: HS Lee. Project administration: YC Bae, SB Nam. Visualization: HS Lee. Writing original draft: HS Lee. Writing - review \& editing: YC Bae, SB Nam, CR Yi, JA Yoon. All authors have read and approved the final manuscript.

\section{ORCID}

Hyun Seung Lee https://orcid.org/0000-0002-8916-9325

Yong Chan Bae https://orcid.org/0000-0002-0268-4667

Su Bong Nam https://orcid.org/0000-0002-9661-0879

Chang Ryul Yi https://orcid.org/0000-0002-4633-0043

Jin A Yoon https://orcid.org/0000-0001-5762-0559

Joo Hyoung Kim https://orcid.org/0000-0002-4893-3761

\section{REFERENCES}

1. Kataru RP, Cuzzone DA, Mehrara BJ. Pathology of lymphedema. In: Gurtner GC, Neligan PC, editors. Plastic surgery e-book. Volume 1. 4th ed. London: Elsevier Health Sciences; 2017. p. 515-28.

2. Boyages J, Kalfa S, Xu Y, et al. Worse and worse off: the impact of lymphedema on work and career after breast cancer. Springerplus 2016;5:657.

3. Sosin M, Yin C, Poysophon P, et al. Understanding the concepts and physiologic principles of lymphatic microsurgery. J Reconstr Microsurg 2016;32:571-9.

4. Matsuura Y, Kawagoe T, Toki N, et al. Long-standing complications after treatment for cancer of the uterine cervix: clinical significance of medical examination at 5 years after treatment. Int J Gynecol Cancer 2006;16:294-7.

5. McLaughlin SA, Wright MJ, Morris KT, et al. Prevalence of lymphedema in women with breast cancer 5 years after sentinel lymph node biopsy or axillary dissection: objective measurements. J Clin Oncol 2008;26:5213-9.

6. Kim K, Kim IJ, Pak K, et al. The feasibility of quantitative parameters of lymphoscintigraphy without significant dermal backflow for the evaluation of lymphedema in post-operative patients with breast cancer. Eur J Nucl Med Mol Imaging 2020;47:1094-102.

7. Bozkurt M, Palmer LJ, Guo Y. Effectiveness of decongestive lymphatic therapy in patients with lymphedema resulting from breast cancer treatment regardless of previous lymphedema treatment. Breast J 2017;23:154-8.

8. Patel KM, Manrique O, Sosin M, et al. Lymphatic mapping and lymphedema surgery in the breast cancer patient. Gland Surg 2015;4:244-56.

9. Patel KM, Lin CY, Cheng MH. From theory to evidence: long-term evaluation of the mechanism of action and flap 
integration of distal vascularized lymph node transfers. J Reconstr Microsurg 2015;31:26-30.

10. Seth AK, Vargas CR, Chuang DJ, et al. Readability assessment of patient information about lymphedema and its treatment. Plast Reconstr Surg 2016;137:287e-295e.

11. Fahradyan A, El-Sabawi B, Patel KM. Understanding patient expectations of lymphedema surgery. Plast Reconstr Surg 2018;141:1550-7.

12. Narushima M, Yamamoto T, Ogata F, et al. Indocyanine green lymphography findings in limb lymphedema. J Reconstr Microsurg 2016;32:72-9.

13. Chang DW, Suami H, Skoracki R. A prospective analysis of 100 consecutive lymphovenous bypass cases for treatment of extremity lymphedema. Plast Reconstr Surg 2013;132: 1305-14.

14. DiSipio T, Rye S, Newman B, et al. Incidence of unilateral arm lymphoedema after breast cancer: a systematic review and meta-analysis. Lancet Oncol 2013;14:500-15.

15. Tsai RJ, Dennis LK, Lynch CF, et al. The risk of developing arm lymphedema among breast cancer survivors: a metaanalysis of treatment factors. Ann Surg Oncol 2009;16: 1959-72.

16. Nielsen I, Gordon S, Selby A. Breast cancer-related lymphoedema risk reduction advice: a challenge for health profes- sionals. Cancer Treat Rev 2008;34:621-8.

17. American Cancer Society. Breast cancer facts \& figures 2013-2014. Atlanta: American Cancer Society; 2013.

18. Petrek JA, Senie RT, Peters M, et al. Lymphedema in a cohort of breast carcinoma survivors 20 years after diagnosis. Cancer 2001;92:1368-77.

19. Ciudad P, Sabbagh MD, Agko M, et al. Surgical management of lower extremity lymphedema: a comprehensive review. Indian J Plast Surg 2019;52:81-92.

20. Maclellan RA, Couto RA, Sullivan JE, et al. Management of primary and secondary lymphedema: analysis of 225 referrals to a center. Ann Plast Surg 2015;75:197-200.

21. Mihara M, Hara H, Araki J, et al. Indocyanine green (ICG) lymphography is superior to lymphoscintigraphy for diagnostic imaging of early lymphedema of the upper limbs. PLoS One 2012; 7:e38182.

22. Mikami T, Hosono M, Yabuki Y, et al. Classification of lymphoscintigraphy and relevance to surgical indication for lymphaticovenous anastomosis in upper limb lymphedema. Lymphology 2011;44:155-67.

23. Yoon JA, Shin MJ, Shin YB, et al. Correlation of ICG lymphography and lymphoscintigraphy severity stage in secondary upper limb lymphedema. J Plast Reconstr Aesthet Surg 2020;73:1982-8. 